\title{
Development of New Electrons Sources for coil-EEFL Tube
}

\author{
Lyuji Ozawa \\ Japanese Government licensed Consultant in Science, Changping Qu, Beijing, China
}

\section{Email address:}

Rotsun4@hotmail.com

\section{To cite this article:}

Lyuji Ozawa. Development of New Electrons Sources for coil-EEFL Tube. Science Research. Vol. 3, No. 4, 2015, pp. $220-229$.

doi: 10.11648/j.sr.20150304.20

\begin{abstract}
After the critical study on the established FL tubes, it has found a formation of the new internal DC electric power generator in the Ar gas space between the needle cathode and anode. The internal DC electric power generator, which completely isolates from the external DC electric circuit by the electron flow, forms with the volumes of the glow light in front of the needle electrodes that are covered with the layer of the frit glass. The lights are generated by the moving electrons between the cathode and anode of the internal DC electric power generator. The results lead us to the development of the prototype of the coil-EEFL tube that has zero power consumption of the external DC driving circuit.
\end{abstract}

Keywords: Green Energy, Power Consumption, Light Source, FL Tube, PDP

\section{Introduction}

The performances of the commercial HCFL tubes have commonly evaluated with the luminous efficiency $\left(\mathrm{lm} \mathrm{W}^{-1}\right)$ for more than 80 years $[1,2]$. However, the luminous efficiency is for the study on the colorimetry and is not use for the evaluation of the light sources. The light sources should be evaluated by either illuminance $\left(1 \mathrm{~m} \mathrm{~m}^{-2}\right)$ or luminance $\left(\mathrm{cd} \mathrm{m}^{-2}\right)$ and irradiance $\left(\mathrm{W} \mathrm{m}^{-2}\right)$ [3]. The determination of either one uses the Ulbricht Sphere. It has found that the Ulbricht Sphere that is widely used has deliberately and wrongly modified for the determination of the luminous efficiency $\left(\mathrm{lm} \mathrm{W}^{-1}\right)$ [3]. The erroneous evaluation of the established FL tubes by the luminous efficiency $\left(1 \mathrm{~m} \mathrm{~W}^{-1}\right)$ had reached a conclusion as the mature technology of the FL tubes on early 1970s. According to the conclusion, the systematic study on the development of the FL tubes had terminated in USA. The American Institute of Physics published the summary of "the basic data of plasma physics" from American Vacuum Society Classics on 1993 [4]. We have carefully studied the established technologies from the above publications. However we cannot find a room for further scientific study on the FL tubes. So far as the study is made with the publications, one may have the same conclusion that the commercial FL tubes are produced with the mature technology.

We have studied the lighted FL tube with the different views from the published information. Main problems in the study on FL tubes and plasma display panel (PDP) are the electron source (cathode) and electron collection source (anode). The FL lamp tube, the lighting cells of the PDP, and other vacuum devices, such as cathode ray tubes (CRT) and vacuum (radio) tubes, use the moving electrons in vacuum and in the vacuum containing the gases in a low gas pressures. In the vacuum devices, the cathode supplies the moving electrons into vacuum and the anode collects arrived electrons from the vacuum. In solids, the anode electrode supplies electrons into the solids and cathode electrode corrects the electrons from the solids. The electric circuits close with the electron flow between cathode and anode, but the moving direction differs with the solids and vacuums. In vacuum, the electrons move from the cathode to the anode. We have found the incomplete electric circuit in the study on the FL tubes and PDP. They take the care the cathode as the electron source in their devices, but no one take care the anode that collects the electrons arrived in their vacuum devices. The electric circuit in the vacuum of their devices is not closed with the collection of the electrons at the anode. Their considerations on the discussion of the FL tubes and PDP are made with the cathode that emits the electrons in the inert $\mathrm{Ar}$ and $\mathrm{Ne}$ gases. They never discuss about the collection of the electrons from the Ar and Ne gas space. Their studies are made with the uncompleted electric circuit. Furthermore, the cathode does not directly inject the electrons into the gas space. Here remains a large room for the study on the FL tube and PDP. We have studied the 
subject of the formation of the cathode and anode in the Ar gas space for the unambiguous solutions of the formation of the internal electric circuits in the Ar gas space.

\section{Closeness of Electrodes by Electron-Flow in Vacuum Less Than $10^{-3} \mathrm{~Pa}$}

We have found the totally different operation mechanisms of the unlighted and lighted FL tubes and PDP. We have found that the ambiguousness of the established FL tubes and PDP comes from the electron source (cathode) and electron collection source (anode) in the Ar gas space in the FL tube. The PDP uses Ne gas space. Ar and Ne atoms are inert gases, hereafter Ar gas. The Ar gas space in the vacuum-sealed glass tube fills with the inert Ar atoms. The inert Ar gas space in unlighted FL tube is electric insulator. The Ar gas space in the lighted FL tubes suddenly changes to the superconductive vacuum for moving electrons [5]. The electrons in the lighted FL tubes move on the superconductive vacuum from the cathode to the anode in the Ar gas space. We must disambiguate the closeness of the electric circuit in the Ar gas space of the lighted FL tube and PDP.

We have studied the basics of the electron flow from the needle cathode to the plate anode in vacuum and in Ar gas space. Figure 1 illustrates the arrangement of the experiments by the needle cathode and plate anode. Figure 1 (A) illustrates the experiments in the vacuum at the pressures less than $10^{-3}$ $\mathrm{Pa}\left(<10^{-5}\right.$ Torr $)$. Figure 1 (B) illustrates the experiments in the Ar gas at the gas pressures $133 \mathrm{~Pa}$ (=1 Torr).

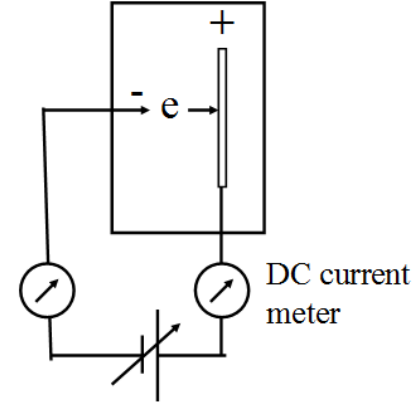

variable DC power

(A) in vacuum

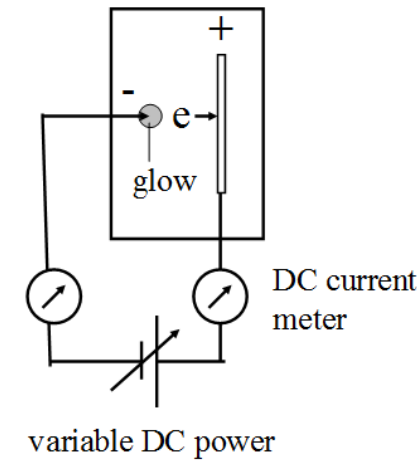

(B) in Ar gas space
Figure 1. Arrangement of experiments by needle cathode electrode and plate anode electrode in vacuum pressure less than $10^{-3} \mathrm{~Pa}$ (A) and in $\mathrm{Ar}$ gas pressure at $1 \mathrm{~Pa}$.

The top of the needle cathode electrode is shaper than $1 \mathrm{x}$ $10^{-6} \mathrm{~m}$. The sharp point of the needle electrode emits the electrons into the vacuum at the pressures lower than $10^{-3} \mathrm{~Pa}$ $\left(<10^{-5}\right.$ Torr) under the application of the DC voltage above $10 \mathrm{~V}$. The amount of the emitted electrons from the cathode and amount of the collected electrons by the anode have confirmed by the DC electric current meters, respectively. The anode collects the same amount of the electrons from the cathode. The detected DC electric currents (I) are linearly changed with the applied voltages (V) with the relation of $\mathrm{V}$ $=\mathrm{IR}$ where $\mathrm{R}$ is electric resistance in the needle and plate electrodes. The DC electric circuit is closed with the flowing electrons in the superconductive vacuum between needle cathode and plate anode. The superconductive vacuum does not have $\mathrm{R}$. The metal electrodes have $\mathrm{R}$. The needle cathode has a limitation with the emitted electrons. The needle metal electrode has the electric resistance $(\mathrm{R})$ that generates the Joule Heat $\left(I^{2} R\right)$. The sharp point of the needle cathode has the high density of the electric current. The sharp point of the needle cathode selectively heats up to a high temperature by the Joule Heat. Consequently, the sharp point of the needle cathode is rounded at the high electric current density. The emitting voltage from the rounded cathode electrode shifts to the high voltages.

The injected electrons into the high vacuum do not generate the light with the reason that the moving electrons do not have a chance to meet atom that floats in the vacuum. The results clearly indicate that the high vacuum provides the superconductive vacuum for the moving electrons. This is well known in the study on CRT.

\section{Needle Cathode in Ar Gas Pressures Higher Than 1 Pa}

As the needle cathode electrode and plat anode electrode set in the $\mathrm{Ar}$ gas space at the $1 \mathrm{~Pa}\left(=10^{-2}\right.$ Torr $)$, the needle cathode does not emit the electron into the Ar gas space by the applied DC voltages below $1 \mathrm{kV}$. The Ar gas space differs from the high vacuum. We must figure out the difference between the high vacuum and Ar gas space. The large number of the inert Ar atoms in the vacuum-sealed glass tube float in the vacuum with the Maxwell-Boltzmann distribution. The average separation distance of Ar atoms is calculated from the Boyle-Charles law and Avogadro's number $\left(6.0 \times 10^{23} \mathrm{~mole}^{-1}\right)$. The average separation distance between Ar atoms is $2 \times 10^{-7} \mathrm{~m} \mathrm{[5].} \mathrm{The} \mathrm{conditions} \mathrm{between}$ Ar atoms have overlooked in the study on the FL tubes and PDP.

The vacuum between Ar atoms fills up with the negative field from the orbital electrons of neighboring Ar atoms. The presence of the negative field in the vacuum between Ar atoms is well known in the analytical chemistry that measures the absorption spectrum of the Ar atoms with a high resolution spectrometer. The absorption spectrum of the Ar atoms at $133 \mathrm{~Pa}$ (=1 Torr) consists with the many sharp lines that attribute to the split energy levels under the electric fields, The split energy levels to the sublevels is caused by the electric field that is the Stark Effect. The splitting of the energy levels to the sublevels is the direct evidence of the presence of the negative electric field in the vacuum between Ar atoms.

The electrons from the sharp point of the needle cathode cannot step in the negative electric field by the Coulomb's repulsion. The neutralization of the negative field is the prerequisite condition that the electrons from the needle 
cathode step in the vacuum between Ar atoms. The neutralization of the negative electric field in the vacuum between Ar atoms is made by the formation of the volume of the ionized Ar atoms at around the top of the needle cathode. The electric field from the sharp point of the needle electrode suddenly ionizes the Ar atoms at nearby the electrode. The ionized Ar atoms form the volume in the diameter of $1 \times 10^{-3}$ $\mathrm{m}$. The volume of the ionized Ar atoms contains the ionized Ar atoms $\left(\mathrm{Ar}^{1+}\right)$, free electrons (e), excited $\mathrm{Ar}$ atoms $\left(\mathrm{Ar}^{*}\right)$, and $\mathrm{Ar}$ atoms. The $\mathrm{Ar}^{1+}$, free electrons, and $\mathrm{Ar}$ atoms are invisible by the naked eyes. Only Ar* emits the sky-blue lights that are the visible lights by the naked eyes. We may monitor the volume of the ionized $\mathrm{Ar}$ atoms at around the needle electrodes by the visible lights that have called as the glow light. The major particles in the volume of the glow light are $\mathrm{Ar}^{1+}$ and free electrons. There is a threshold voltage for the formation of the volume of the glow light. The threshold voltage is $1 \mathrm{kV}$, independent on the Ar gas pressures below $1 \times 10^{4} \mathrm{~Pa}\left(\approx 10^{2}\right.$ Torr $)$. The presence of $\mathrm{Ar}^{1+}$ in the inside of the volume of the glow light neutralizes the negative field in the vacuum between Ar atoms.

The electrons in the volume of the glow light may move on in the neutralized vacuum under the vector electric field, $\mathrm{F}_{\text {vect }}$, between the cathode and anode in the glass tube. The free electrons in the volume of the glow light move to the direction of the anode under the $F_{\text {vect }}$. The moving electrons are accelerated by the $F_{\text {vect }}$. The accelerated electrons in the volume of the glow light may step in the Ar gas space at the nearby the volume of the glow light. The stepped electrons from the volume of the glow light may meet the Ar atom and ionizes it. The ionized $\mathrm{Ar}^{1+}$ neutralizes the vacuum between Ar atoms at nearby the volume of the glow light. The volume of the glow light at around the cathode needle electrode becomes the new electron supplier (cathode) in the Ar gas space. The neutralization of the negative filed in the Ar gas space instantly propagates to the entire Ar gas space with the moving speed of the electrons in the Ar gas space under the $F_{\text {vect. }}$ The moving speed of the electrons in the Ar gas space is calculated as $10^{5} \mathrm{~m}$ per second that is much shorter than the moving speed of $10^{8} \mathrm{~m}$ per second of the electrons in the vacuum. The slow speed is caused by the scattering of the moving electrons with the $\mathrm{Ar}$ atoms. The subsequent electrons from the cathode move on in the neutralized vacuum between Ar atoms toward to the plate anode. The glass tube instantly and continuously lights up within $10^{-5}$ sec by the moving electrons between new cathode and anode. Hence, the volumes of the glow light on the needle cathode works as the new electron source in the Ar gas space, instead of the sharp point of the needle electrode. The anode plate electrode works as the collector of the arrived electrons.

Since the electrons from the volume of the glow light on the needle cathode move on in the Ar gas space toward to the plate anode, the sharp point of the needle cathode anymore directly inject the electrons into the Ar gas space. Everywhere of the needle cathode electrodes supply the electrons to the volume of the glow light. Consequently, the sharp point of the needle cathode electrode do not rounded by the Joule Heat at the high electric current, e. g., $0.5 \mathrm{~mA}$. This is the advantage of the volume of the glow light as the electron source in the Ar gas space. The size of the volume of the glow light at around the needle cathode does not change with the Ar gas pressures up to 13,300 Pa (=100 Torr). The supplement of the electrons from the needle cathode and the collection of the plate anode have confirmed from the DC current meters. The detected currents are the same.

\section{Invalided Avalanche Model of Moving Electrons in Ar Gas Space}

With the arrangement of the needle cathode and the plate anode, we have observed the followings. As the vacuum sealed glass tube contains the Ar gas at the pressures higher than $1 \mathrm{~Pa}$ ( $=10^{-2}$ Torr), the emitted lights by the Ar* gradually spread from the longitudinal direction in the Ar gas space in the glass tubes. The spreading of the emitted lights from the longitudinal direction is widening with the distance from the needle cathode, as illustrated in Figure 2 (A). The spreading of the lights is caused by the excitation of the Ar atoms $\left(\mathrm{Ar}^{*}\right)$ by the scattered electrons from the longitudinal direction under $F_{\text {vect }}$. The scattering of the moving electrons is by the Coulomb's repulsions that is the electric field of the orbital electrons $\left(\mathrm{F}_{\text {orb }}\right)$ in the floating Ar atoms. By the Coulomb's repulsion from the $\mathrm{F}_{\text {orb}}$, the $\mathrm{Ar}$ atoms becomes to $\mathrm{Ar}^{1+}$ with one free electron. The moving electrons in the gas space are the invisible by the eyes. The spreading of the electrons in the Ar gas space is monitored by the emitted sky-blue lights from the Ar*.

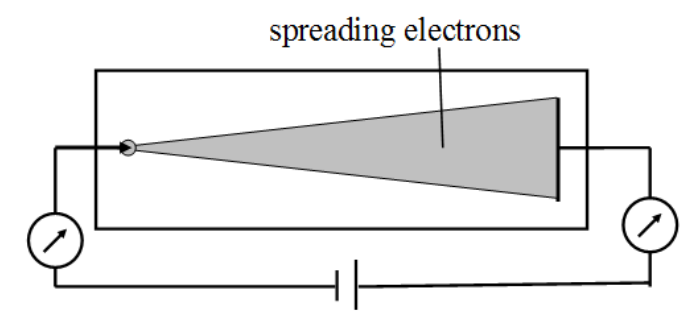

(A) combination of needle and plate electrodes

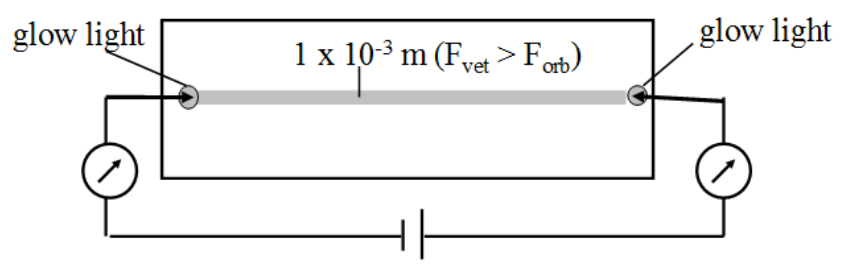

(B) combination of needle electrodes

Figure 2. Lighted path of excited Ar atoms by combination of needle cathode electrode and plate anode electrode (A) and combination of the needle cathode and anode (B).

The spread of the lights in the Ar gas space from the longitudinal direction is widened with the distance from the cathode. At the given distance from the cathode, the lights widely spread with the Ar gas pressures. The widening of the spreading lights had been explained by the avalanche of the moving electrons in the Ar gas space $[1,2]$. In reality, the 
spreading of the lights is the scattered electrons by the Coulomb's repulsion from the orbital electrons of the Ar atoms that are uniformly floating in the entire vacuum by the Maxwell-Boltzmann distribution. The spreading of the lights is not caused by the avalanche. The plate anode is used for the collection of all scattered electrons in the Ar gas space. This is the reason that the vacuum glass tube contains the anode plate shown in Figure 2-(A). In the contrary to the expectation of the avalanche model, the numbers of the collected electrons by the anode plate coincide with the number of the electrons from the needle cathode. The electrons never multiply by the avalanche (spreading) in the Ar gas space. The spreading lights in the Ar gas space have confirmed by the following experiments.

As the needle anode, instead of the plate anode, was used, the needle anode was also covered with the volume of the glow light. The volume of the glow light on the needle anode acts as the new anode in the Ar gas space. The new cathode and anode electrodes formed the strong line-like longitudinal $\mathrm{F}_{\text {vect }}$ in the Ar gas space in the vacuum-sealed glass tube. The scattered electrons by the $\mathrm{F}_{\text {orb }}$ of the Ar atoms immediately take the strong and narrow longitudinal $\mathrm{F}_{\text {vect. The following }}$ experiments were made with the Ar gas pressure at $133 \mathrm{~Pa}$. As the $F_{\text {vect }}>F_{\text {orb }}$, the lights in the Ar gas space do not widely spread in the Ar gas space, as illustrated in Figure 2 (B). One may observe the straight line like of the lights in the Ar gas space in the glass tubes. The widths of the emitted lines are narrowing with the high applied voltage to the electrodes: $F_{\text {vect }}>F_{\text {orb }}$. The widths of the lighted line are widened with the high Ar gas pressures under the given applied voltage.

\section{Formation of Internal DC Electric Power Generator in Ar Gas Space}

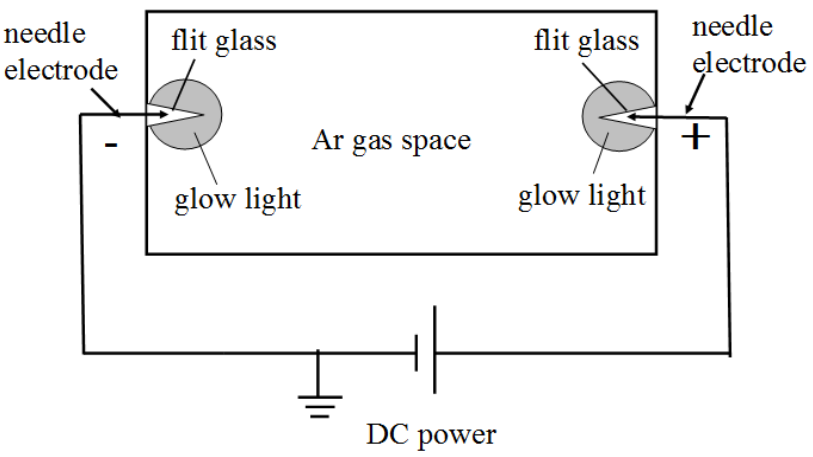

Figure 3. Schematic illustration of needle cathode and anode electrodes covered with layer of frit lass in Ar gas space. Frit glass layers are covered with volume of glow lights under application of DC electric potential to needle electrodes.

It has found that the volumes of the glow light on the needle electrodes form the new cathode and anode in the Ar gas space. Then, we are intent on the elimination of the DC electric power consumption at the external DC electric device. As the entire surfaces of the needle electrodes were covered with the thin layer (less than $1 \times 10^{-4} \mathrm{~m}$ ) of the frit glass that is the electric insulator. The electrons from the needle electrodes cannot penetrate through the frit glass layer. There are two kinds of the frit glasses; (a) crystallized frit glass and (b) amorphous frit glass. We took the crystallized frit glass for a security of the pine hole in the flit glass layer. We had surely detected the individual glow light at around needle cathode and anode that are covered with the layer of the frit glass. The volume of the glow lights appeared at around the electrodes with the application of the DC voltages higher than $1 \mathrm{kV}$. Figure 3 illustrates the configuration of the experiments. The glow lights start at around needle electrodes that are covered with the layer of the frit glass. The entire area of the frit glass layer was covered with the volume of the glow light with the thickness around a few $\mathrm{mm}$. The sharp point of the needle electrodes is the necessary condition for the start of the ionization of the Ar atoms by the electric field from the top of the needle electrodes.

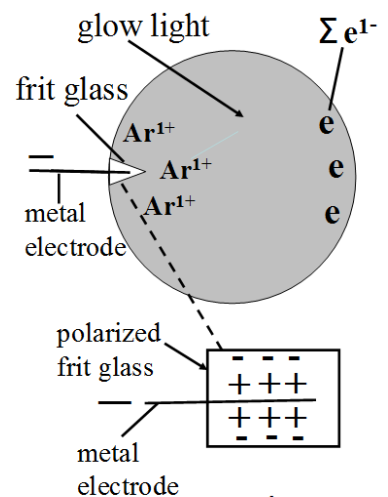

(A)

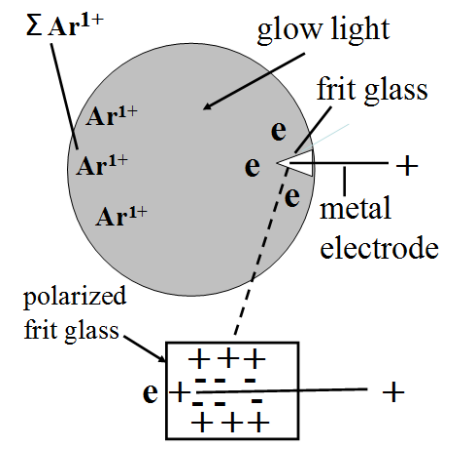

(B)
Figure 4. Explanation of mechanisms of formation of internal DC electric power generator in Ar gas space in vacuum-sealed glass tube.

Figure 4 illustrates the details of the formation of the volume of the glow light on the polarized frit glass layer that covers the surfaces of the needle cathode and anode. The crystallized thin frit glass layer on the needle anode is polarized by the electric field from the needle anode. The Ar atoms in front of the polarized frit glass layer are ionized by the electric field of the polarized thin frit glass layer. Figure 4 (A) explains the distribution of the electrons and $\mathrm{Ar}^{1+}$ in front of the frit glass layer on the negative needle electrode. The $\mathrm{Ar}^{1+}$ in the volume of the glow light is attracted by the negative charges in the polarized frit glass. The attracted $\mathrm{Ar}^{1+}$ stays in front of the negative charges of the polarized frit glass. As the consequence, other side of the volume of the glow light has many free electrons $\left(\Sigma \mathrm{e}^{-}\right)$. The volume of the glow light on the frit glass of the positive needle electrode has many $\mathrm{Ar}^{1+}$ $\left(\Sigma \mathrm{Ar}^{1+}\right)$, as illustrated in Figure 4 (B). Thus the electric charges are unevenly distributed in the volume of the glow lights by the electric field from the polarized insulator. The $\Sigma \mathrm{e}^{-}$ on the volume of the glow light at the left side forms the cathode, and the $\Sigma \mathrm{Ar}^{1+}$ in the volume of the glow light at the right side forms the anode. Hence the combinations of $\Sigma \mathrm{e}^{-}$and $\Sigma \mathrm{Ar}^{1+}$ in the volume of the glow lights form the electrodes of the internal DC electric power generator in the inside of the Ar gas space under the assistance of the electric fields from the needle electrodes. Hence the internal DC electric power 
generator forms in the inside of the Ar gas space in the vacuum sealed glass tube, without the supply and collection of the electrons by the needle metal electrodes. No supplement and collection of the needle electrodes have confirmed by the no DC current at the needle electrodes.

By the internal DC electric power generator, the electrons from the cathode move on in the superconductive vacuum between $\mathrm{Ar}$ atoms toward to the anode [5]. The moving electrons in the Ar gas space meet the Ar atoms. The moving electrons ionize and excite the Ar atoms. The moving electrons generate the lights from the Ar gas space. The anode collects the electrons from the Ar gas space. The electrons at the anode recombine with the $\mathrm{Ar}^{1+}$ to return to $\mathrm{Ar}$ atoms. The internal DC electric power generator reserves the Ar atoms in the operation. Furthermore, the electric power consumption of the internal DC electric power generator completely isolates from the electric power consumption of the external DC driving circuit. The both electric circuits are conjugated with the electric field from the electrodes of the external driving circuit, without the electron flow. The conjugation of the electric field does not consume the electric energy. The external DC driving circuit does not have the DC current in the lighted glass tube.

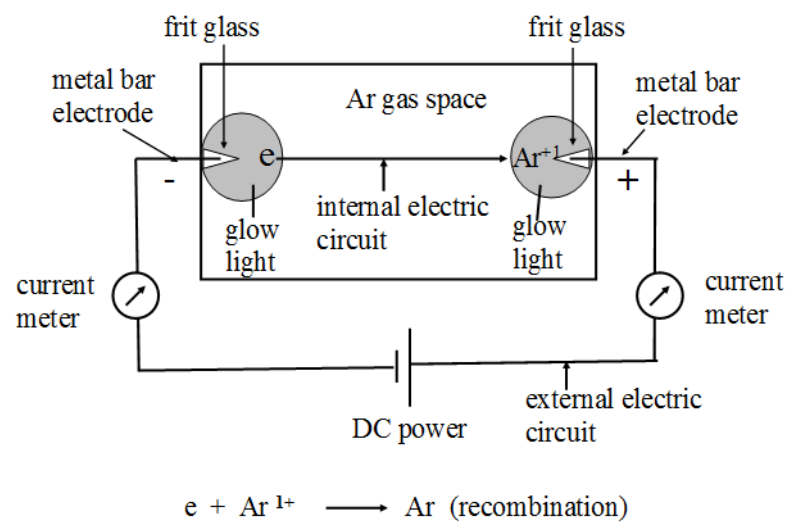

Figure 5. Explanation of active internal DC electric power generator in Ar gas space in vacuum-sealed glass tube.

Hence, we have found the coexistence of (a) the internal DC electric power generator (electric circuit) in the Ar gas space and (b) external DC electric circuit in the vacuum-sealed glass tube, as illustrated in Figure 5. The electrons from the cathode move on in the superconductive vacuum between Ar atoms in the Ar gas space toward to the anode of the internal DC power generator. The moving electrons in the Ar gas space meet the $\mathrm{Ar}$ atoms. The moving electrons ionize the $\mathrm{Ar}$ atom $\left(\mathrm{Ar}^{1+}\right)$ with free electrons $\left(\mathrm{e}^{-}\right)$and excite the Ar atoms $\left(\mathrm{Ar}^{*}\right)$. The moving electrons, $\mathrm{Ar}^{1+}$, and free electrons, and Ar atoms are invisible by the naked eyes that are the abstraction in the brain. The Ar* emits the visible sky-blue lights in the vacuum-sealed glass tube that is concrete material. We may use the visible sky-blue lights as the monitor of the existence of the moving electrons, $\mathrm{Ar}^{1+}$, free electrons, and $\mathrm{Ar}$ atoms in the $\mathrm{Ar}$ gas space of the vacuum-shielded glass tube. The energy of the external DC driving circuit does not involve in the generation of the lights in the vacuum-sealed glass tube. The external driving circuit and internal DC electric circuit coexist in the operation of the lighting of the glass tube that contains the Ar gas at the pressures higher than $1 \mathrm{~Pa}$. The external driving circuit and internal DC electric circuit have the different role in the operation of the lighted vacuum-sealed FL tubes and the lighting cells of the PDP. This is the most important points for the study on the FL tubes and PDP. The moving electrons in the internal DC electric circuit exclusively involve in the generation of the lights in the vacuum-sealed glass tube. The external driving circuit assists the holding the internal DC electric circuit in the Ar gas space by the electric field from the needle electrodes. The power consumption of the external driving circuit does not involve in the generation of the lights in the vacuum-sealed lighting FL tubes and cells of the PDP.

The needle electrodes in the vacuum-sealed glass tube, which are covered with the frit glass layers, certainly have the both cathode and anode in the inside of the Ar gas space. By the eyes observation, the entire Ar gas space uniformly lights up with the sky-blue light, indicating that the electrons surely move on in the vacuum between cathode and node of the internal DC electric power generator. In the internal DC electric power generator, the volume of the glow light as the cathode supplies the electrons into the vacuum between $\mathrm{Ar}$ atoms. The electrons arrived to the glow light on the anode recombine with the $\mathrm{Ar}^{1+}$ in the volume of the glow light as the anode. The recombinations of the electrons and $\mathrm{Ar}^{1+}$ in the volume of the glow light as the anode return to Ar atoms. The returned Ar atoms again ionize by the electric field from the electrodes of the external driving circuit. The Ar atoms in the vacuum-sealed glass tubes reserve in the operation of the lighted glass tubes.

The scattered electron by the monitored with the excitation of the $\mathrm{Ar}^{*}$ has the kinetic energy for the excitation energy of Ar*. The simply calculated ratio of the numbers of the $\mathrm{Ar}^{1+}$ to the numbers of Ar* in the lighted Ar gas space is around 62 to $1\left(=1000 \mathrm{~V} \times 16^{-1} \mathrm{~V}\right)$. The large numbers of the $\mathrm{Ar}^{1+}$ heat up the Ar gas space by the change in the entropy.

The FL tube does not have the heating device. The origin of the lights of the lighted FL tubes is the excited $\mathrm{Hg}$ atoms $\left(\mathrm{Hg}^{*}\right)$ in the Ar gas space. The $\mathrm{Hg}$ atoms in the $\mathrm{Ar}$ gas space evaporate from the $\mathrm{Hg}$ droplets on the phosphor screen. The Ar gas space in the FL tubes only heats up the temperatures by the ionization of the Ar atoms by the change in the entropy. The high temperatures of the Ar gas space of the lighted FL tubes are obtained with the high Ar gas pressures as possible in the lighted FL tubes. This is the reason that the FL tubes use the Ar gas, instead of $\mathrm{Ne}$ gas, in order to evaporation of a large amount of the $\mathrm{Hg}$ atoms from the $\mathrm{Hg}$ droplets on the phosphor screen. You never add other gases, like as $\mathrm{Ne}$ and $\mathrm{Kr}$, into the Ar gas of the practical FL tubes.

\section{Superconductive Vacuum Between Ar Atoms in Ar Gas Space}

The electrons from the volume of the glow lights move on in the vacuum between Ar atoms in the Ar gas space. The 
separation distance between $\mathrm{Ar}$ atoms in the FL tubes is calculated as $2 \times 10^{-7} \mathrm{~m}$ that is very wide vacuum space for the moving electron in the diameter of $5.6 \times 10^{-13} \mathrm{~m}$. Each floating Ar atom thermally vibrates in the vacuum space in the limited space in $10^{-10} \mathrm{~m}$. The floating Ar atoms in the vacuum in FL tubes do not have the influence of the thermal perturbation from the neighboring Ar atom. No thermal perturbation of the floating Ar atoms can be confirmed by the measurements of the optical absorption lines by the high resolution spectrometer. You may obtain the sharp absorption lines that indicate no thermal perturbation from the neighbor Ar atoms. If the energy level is thermally perturbed from the neighbor atoms, you may detect the absorption bands. It can say that the wide vacuum $\left(=2 \times 10^{-7} \mathrm{~m}\right)$ between $\mathrm{Ar}$ atom provides the superconductive vacuum for the moving electrons. The moving electrons in FL tubes do not have the thermal perturbation from the neighbor atoms. The thermal perturbation gives the electric resistance to the moving electrons. The moving electrons lose the kinetic energy by the Joule Heat. The moving electrons in the FL tubes do not have the electric resistance. The atoms in the solids are arranged at the lattice sites that have $\sim 10^{-10} \mathrm{~m}$ separation. The solids in the film have the absorption bands in the detected spectrum. The moving electrons in the solids inevitably have the electric resistance that generates the Joule Heat. It has believed for 80 years that the moving electrons in the Ar gas space of the FL tubes have the electric resistances $[1,2,4]$ with the analogy of the solids. No electric resistance in the vacuum between the Ar atoms of the experimental glass tube holds the constant voltage at $1 \mathrm{kV}$, even though the application of the voltages higher than $1 \mathrm{kV}$ to the electrodes of the external DC electric circuit, like as the voltage regulation tube [6]. With other word, the Ar gas space provides the superconductive vacuum, that give rise to no potential change in the Ar gas space by IR $=\mathrm{V}$. The upper limitation of the applied voltage comes from the arc current in the Ar gas space of the internal DC electric generator. Above $0.5 \mathrm{~mA}$, the DC current generates the strong zigzag line-lights, like as the thunder lighting (arc current).

The extraordinary advantage of the moving electrons in the superconductive vacuum is the quantum efficiency, $\eta_{\mathrm{q}}$. The $\eta_{\mathrm{q}}$ is given by the ratio of the number of the generated visible photons per unit volume of $\operatorname{Ar}$ gas space $\left(\mathrm{m}^{3}\right)$ per unit time (s) by one moving electron in the lighted Ar gas space. The Ar gas in the tested tube contains only Ar atoms. The excited Ar atoms in the tested tube emit the sky-blue lights. The calculated number of the $\eta_{\mathrm{q}}$ is $10^{16}$ photons $\left(\mathrm{m}^{3} \mathrm{~s}\right)^{-1}$. The origin of the lights of the commercial FL tubes is the excited $\mathrm{Hg}$ atoms $\left(\mathrm{Hg}^{*}\right)$ in the $\mathrm{Ar}$ gas space. The number of the evaporated $\mathrm{Hg}$ atoms is $10^{-3}$ times of that of the Ar atoms, corresponding to $\eta_{\mathrm{q}}=10^{13}$ photons $\left(\mathrm{m}^{3} \mathrm{~s}\right)^{-1}$. As the reference, the solid lighting lamp that has the electric resistance has only $\eta_{\mathrm{q}} \approx 0.5$ photons. The lighted FL tubes and the lighting cells of PDP have the unrivaled advantage over the solid lighting devices, like as the LED lamp. The extraordinary advantage of the FL tubes have concealed by the evaluation of the power consumption of the external AC driving circuits.

\section{Operation Conditions by the External Driving Circuit}

As the surface of the needle electrodes is covered with the layer of the frit glass, the external DC power supply to the needle electrodes does not have the electric current. This means that the power consumption of the external DC power supply to the tested lighting tube is zero. The power consumption of the DC power supply is limited with the power consumption of the electric circuit that generates the DC voltages higher than $1 \mathrm{kV}$, hopefully $5 \mathrm{kV}$. The results suggest us that the FL tubes that contain the evaporated $\mathrm{Hg}$ atoms in the Ar gas can be operated with the zero power consumption of the external DC driving device. The light source with the zero electric power consumption may hold the unrivaled potential as the light sources. Unfortunately, we do not have the proper DC power supply with $5 \mathrm{kV}$ in our hand.

We have the following experiments. The volume of the glow light instantly disappears from the Ar gas space as the DC power to the needle electrodes turns off. The volume of the glow lights instantly appear as the DC power turns on. The results suggest us the tested glass tubes with the needle electrodes may operate with the AC driving circuit to the electrodes. Yes, by the observation of the naked eyes, the glass tube constantly emits the sky blue lights under the AC driving circuit that has the frequencies higher than $50 \mathrm{~Hz}$ with the AC applied voltages higher than $2 \mathrm{kV}$. A problem arises as to that the external $\mathrm{AC}$ driving circuit has a large $\mathrm{AC}$ current in 0.05 A. The AC current in the external AC driving circuit increases with the Ar gas pressures. The operation conditions of the testing glass tubes are the similar with the commercial cold cathode FL tubes (CCFL tube) and HCFL tubes. The needle electrodes covered with the frit glass layer pick up the $\mathrm{AC}$ induced current from the $\mathrm{C}_{\text {tube }}$.

The FL tubes use the phosphor screen that is the layers of the phosphor particles. The study on the preparation of the phosphor particles has the long histories more than 100 years. The text books of the phosphor powders describe the practical phosphor powders $[7,8]$. The phosphor powder in $1 \times 10^{-3} \mathrm{~kg}$ $(=1 \mathrm{~g})$ contains $3 \times 10^{15}$ particles. The phosphor powders in $\mathrm{kg}$ orders can be produced by the raw materials in the quartz crucible less than $0.1 \mathrm{~m}^{3}$ in the heated furnace. The produced phosphor particles contain the various shapes and sizes. Furthermore, the produced phosphor particles are not single crystal. The phosphor particles are the anisotropic crystals with the high electric transitions between the excited levels to the grand level [9]. Furthermore, each phosphor particles are the polycrystalline that contain plural growing axes [9]. Figure 6 shows the photograph of the phosphor particles taken by the scanning electron microscope (x 2000). As the polycrystalline phosphor particles can be produced with the well controlled conditions [10], the surfaces of each polycrystalline phosphor particle have the many sharp points and sharp edge-lines. Even though the phosphor particles are the polycrystalline particles, the anisotropic crystals smoothly and largely deform the crystal structure under the electric field. The deformed phosphor particles are the polarized particles. The surfaces of 
the polarized phosphor particles have many sharp edge-lines and sharp points that are the equivalent with the sharp needle electrodes in the Ar gas space. The phosphor screen of the FL tubes is made with the phosphor powder in a few grams that contain $10^{13}$ particles. Therefore the many sharp edge-lines and sharp points of the largely deformed phosphor particles in the screen may generate the strong electric fields to the Ar gas space, resulting in the generation of the large volume of the glow light in front of the polarized phosphor particles.

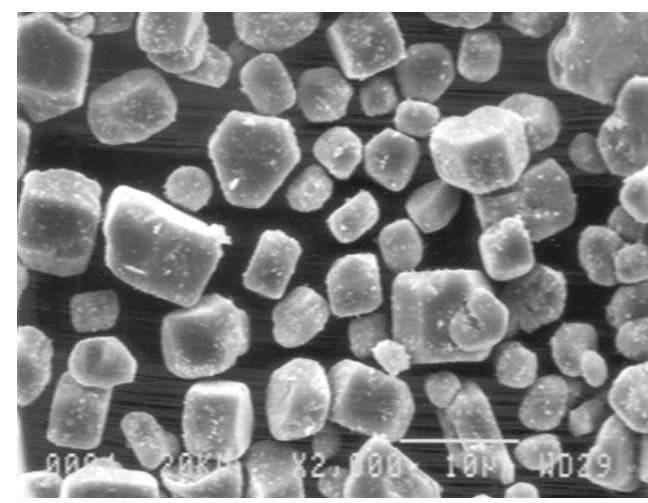

Figure 6. Photograph of scanning electron microscope (x 2000) of phosphor particles used in experiments.

This gives an idea that if the FL tubes with the phosphor screen have the external electrodes (EEs) on the outer glass tube, the EEFL tubes may have the internal DC electric power generator. If it is so, the EEFL tubes emit the brighter lights. The number of the sharp points of the surfaces of the polarized phosphor particles increases with the wideness of the electrodes on the outer glass wall. We have found the EEFL tubes light up with the coil-electrodes on the glass wall, without the metal electrodes in the inside of the FL tubes. In this case, the coil-EEFL tubes may operate with the AC driving circuit with the less AC power consumption. Figure 7 shows the photograph of the lighted coil-EEFL tube with the AC driving circuit with $30 \mathrm{kHz}$. The active power consumption $\mathrm{W}_{\text {act }}$ of the external AC driving circuit is a significantly low as compared with the needle electrodes covered with the layer of the frit glass. The coil-EEFL tubes also operated with the DC electric circuit. For the study on the details of the coil-EEFL tubes, we must clarify the invalided technologies in the established FL tubes for the avoidance of the confusion by the established FL technologies, including PDP technologies.

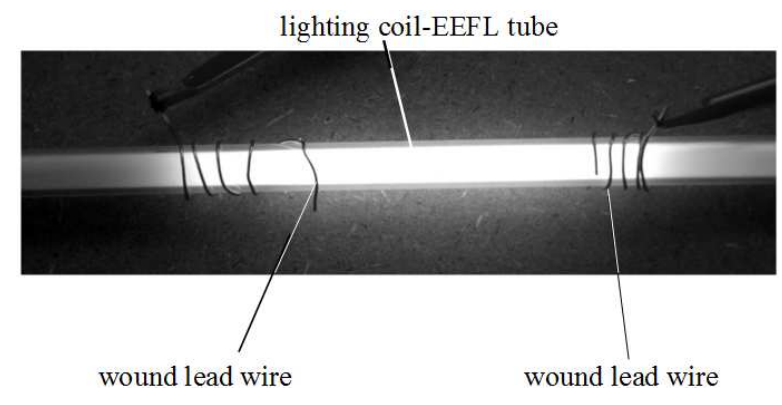

Figure 7. Photograph of lighted coil-EEFL tube as prototype.

\section{Invalided Technologies in Study on Established FL Tubes}

The commercialized FL tubes are called as the hot-cathode FL (HCFL) tubes. The HCFL tubes have developed with many hypotheses and erroneous assignments of the experimental results. Those generate a deadlock of the study of the FL tubes. We cannot use the invalided technical concepts and wrongly defined words for the study on the advanced coil-EEFL tubes.

After the clarification, we will find a large room that remains for the significant improvements of the lighted coil-EEFL tubes as the light source; especially (a) the AC active power consumption, $\mathrm{W}_{\text {act }}$, and (b) illuminance $\left(\mathrm{lm} \mathrm{m}^{-2}\right)$ of the lighted FL tubes. The typical example, as already described, is the $\mathrm{W}_{\text {act }}$ of the external AC driving circuit. The $\mathrm{W}_{\text {act }}$ does not relate to the generation energy of the lights from the lighted FL tubes.

\subsection{Luminous Efficiency $\left(\mathrm{lm} \mathrm{W}^{1}\right)$ is for Colorimetry}

At present, the performance of the lighted FL tubes has evaluated with the luminous efficiency $\left(\operatorname{lm~W}^{-1}\right)[1,2,4]$. The luminous efficiency $\left(\mathrm{lm} \mathrm{W}^{-1}\right)$ is for the study of the colorimetry. The luminous efficiency $\left(1 \mathrm{~m} \mathrm{~W}^{-1}\right)$ never use for the study of the light sources. The light sources should be evaluated with either the luminance $\left(\mathrm{cd} \mathrm{m}^{-2}\right)$ or illuminance $\left(\operatorname{lm~} \mathrm{m}^{-2}\right)$, and irradiance $\left(\mathrm{W} \mathrm{m}^{-2}\right)$, which are determined by the use of the Ulbricht Sphere [3]. The $\mathrm{W}$ of the irradiance $\left(\mathrm{W} \mathrm{m}^{-2}\right)$ is the energy of the lights that determines by the bolometer, The $\mathrm{W}$ of the luminous efficiency $\left(\mathrm{lm} \mathrm{W}^{-1}\right)$ is not the energy of the lights. The $\mathrm{W}$ of the luminous efficiency has determined by the $\mathrm{W}_{\text {tube }}$ at the electrodes of the FL tube. By the evaluation of the FL tubes by the erroneous luminous efficiency $\left(\mathrm{lm} \mathrm{W}^{-1}\right)$, the compact HCFL tubes made in China have totally lost the credibility from the USA customers on before 2005 with a short operation life less than 500 hours. The HCFL tubes made in USA usually have the operation life longer than 2000 hours. The recovery of the lost credibility from the users is very hard. Why the compact HCFL tubes made in China reduced the Ar gas pressures to the $133 \mathrm{~Pa}$ (=1 Torr) from $931 \mathrm{~Pa}$ (=7 Torr) of the regular HCFL tubes? The determined $\mathrm{W}$ is decreased with the low $\mathrm{Ar}$ gas pressures for the increase the luminous efficiency $\left(\mathrm{lm} \mathrm{W}^{-1}\right)$, but the $\mathrm{W}$-filament coil rapidly evaporates with the low Ar gas pressures, resulting in the short operation life. The FL tubes never evaluate with the luminous efficiency $\left(\operatorname{lm~} \mathrm{W}^{-1}\right)$. The control panel of the Ulbricht Sphere of many lighting laboratories and FL producers in many Asian countries installs the light sensor that determines the lumen (lm) by the improper modification of the Ulbricht Sphere [3]. We take the illuminance $\left(\mathrm{lm} \mathrm{m}^{-2}\right)$ for the evaluation of the lighted FL tubes and lighted coil-EEFL tubes.

\subsection{FL Tube is not Discharge Lamp}

The FL tubes have named as the FL discharge lamp [1, 2, 4]. Accordingly, the commercial FL tubes are named as the FL discharge lamps. Practical FL tubes are not the discharge lamp. The optimized commercial FL tubes contain inert Ar atoms at 
around $930 \mathrm{~Pa}$ (=7 Torr). Ar atoms in the FL tube float in vacuum. The study on the FL tubes belongs to the Vacuum Physics with the low Ar gas pressures that give the $2 \times 10^{-7} \mathrm{~m}$ separation distance between Ar atoms [5]. The electrons move on in the superconductive vacuum in the Ar gas space from the cathode to the anode that is not the discharge of the electrons. The electrons in the solid devices move on from anode and cathode. They never call the solid electric devices as the discharge devices, because the moving electrons are controlled by the electric field between anode and cathode. The moving electrons in the FL tubes are well controlled by the electric field between the cathode and anode of the internal DC electric power generator. The FL tubes are not the discharge lamp. It is FL tubes.

\subsection{No Thermoelectron Emission in HCFL Tubes}

The FL tubes use the moving electrons in the Ar gas space. The moving electrons require the electron supplier as cathode and electron collector as anode. There is the hypothesis of the study of the electron emission and collection of the HCFL tubes. The hypothesis is that (a) the heated $\mathrm{BaO}$ particles on the W-filament coils, as the cathode, in the lighted HCFL tubes directly inject the thermoelectrons into the Ar gas space. And (b) the heated $\mathrm{BaO}$ particles on the $\mathrm{W}$-filament coils at the opposite side collect the arrived electrons as the anode. The hypothesis has a contradiction as the role of the heated $\mathrm{BaO}$ particles. The contradictory of the hypothesis has not scientifically verified yet in the study on the FL tubes. The contradictory of the hypothesis has led the study of (a) the invalided $\mathrm{W}$ of the external $\mathrm{AC}$ driving circuit and (b) the invalided luminous efficiency $\left(\mathrm{lm} \mathrm{W}^{-1}\right)$.

The drilled studies on the thermoelectron emission into the vacuum had made with the development of the cathode-ray tubes (CRT) and vacuum (radio) tubes that belong to the vacuum physics. The $\mathrm{Ba}$ atoms arranged at top layer on the heated $\mathrm{BaO}$ layers on the cathode steadily emit the thermoelectrons into the vacuum at the pressures lower than $10^{-4} \mathrm{~Pa}\left(<10^{-7}\right.$ Torr $)$. The electrically conductive electrode as the anode in CRT and vacuum tubes collects arrived electrons from the vacuum. The operation condition of the devices is the DC electric circuit. There is a strict limitation with the vacuum pressures for the thermoelectron emission from the heated $\mathrm{Ba}$ atoms on the $\mathrm{BaO}$ layers. The heated $\mathrm{Ba}$ atoms on the $\mathrm{BaO}$ layers do not emit the thermoelectrons into the vacuum at the pressures higher than $0.1 \mathrm{~Pa}\left(>10^{-3}\right.$ Torr). The $\mathrm{BaO}$ particles in the poor vacuum instantly become to the electric insulator. The FL tube contains Ar gas pressures higher than $9 \times 10^{2} \mathrm{~Pa}(>$ 7 Torr). The $\mathrm{BaO}$ particles on the W-filament coils in the lighted FL tubes are the electric insulators. The electric insulator never emits the electrons in the Ar gas space. The hypothesis of the thermoelectron emission in the HCFL tubes is invalided by the results of the drilled study on CRTs and vacuum tubes. The electrodes of the HCFL tubes never inject the electrons into the Ar gas space. The HCFL tubes are the nominal HCFL tubes.

If the AC high voltage applies to the electrode, the HCFL tube has the Arc current to the electrode. Figure 8 shows the photograph that the HCFL tube has the arc current to the electrode.

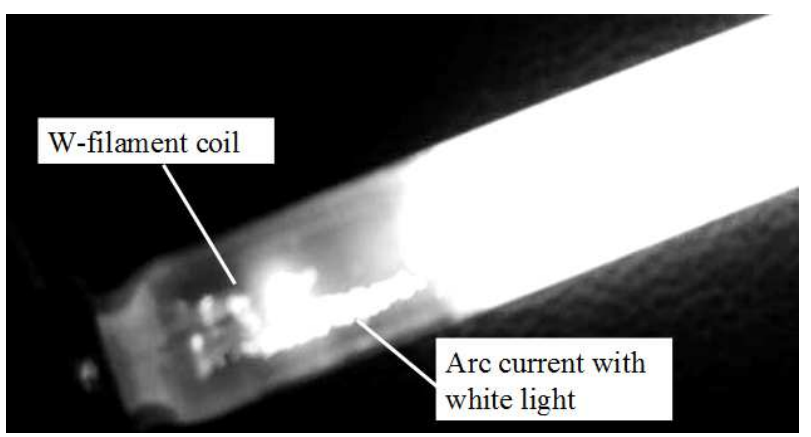

Figure 8. Photograph of Arc current toward to electrode of lighted FL tube.

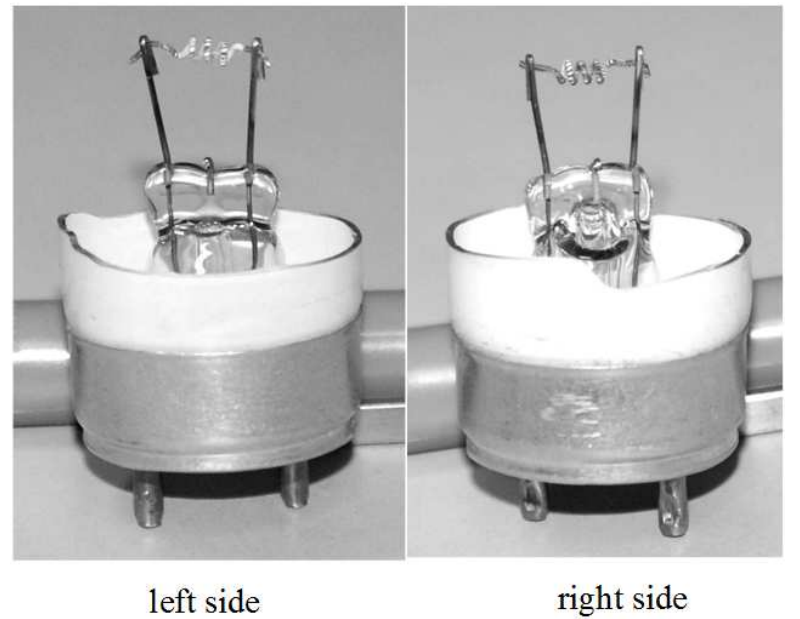

Figure 9. Photographs of the twisted W-filament coils at left side and right side of commercial HCFL tube.

By the erroneous hypothesis, many commercial HCFL tubes use the twisted W-filament coils for the expectation that increases the thermoelectron emission from the heated $\mathrm{BaO}$ particles on the W-filament coils. Figure 9 shows photograph, as the evidence, of the twisted W-filament coils of the commercial HCFL tube that is produced by a leading FL tube producer on the world. The twisted $\mathrm{W}$-filament coils are unnecessary for the FL tubes. The real electron sources of the lighted HCFL tubes are the $4 \mathrm{G}$ electron source [11]. The $4 \mathrm{G}$ electron sources are formed by the volume of the ionized $\mathrm{Ar}$ atoms of the heated $\mathrm{Ar}$ gas at near heated spot on the $\mathrm{W}$-filament coil. The $4 \mathrm{G}$ electrons source requires the initial ionization energy of the Ar gas space and holding energy of the volume of the heated Ar gas space. After the formation of the $4 \mathrm{G}$ electron sources, the holding condition of the $4 \mathrm{G}$ electron source determine the applied voltages to the electrodes of the lighted HCFL tubes. The W-filament coil with $\mathrm{BaO}$ particles never collects the electrons from the Ar gas space. The electrodes of the lighted FL tubes never close with the thermoelectrons from the heated $\mathrm{BaO}$ particles on the W-filament coils. The HCFL tube is the nominal HCFL tube.

\subsection{No AC Current Waveform of Lighted FL Tubes}

The operation of the lighted FL tubes by the AC driving 
circuit have supported by the current waveform detected at the electrodes of the external AC driving circuit of the lighted FL tubes. This is misinterpretation of the detected waveform on the screen of the oscilloscope. In reality, oscilloscope does not have current sensor. The oscilloscope has only the voltage sensor. The oscilloscope cannot directly detect the current waveform in the driving circuit. The waveforms in the lead wire detect the voltage changes in the inserted small resistance a few $\Omega$. The study on the waveforms of the lighted HCFL tubes should be detected at the everywhere of the components of the AC driving circuit for the comprehension of the properties of the electric current, that is the electron flow, in the AC driving circuit.

Figure 10 shows the waveforms on the screen of the oscilloscope that have determined the everywhere of the commercial 40W-HCFL tube with the ballast (chock coil). You may detect the sine wave at everywhere in the driving circuit, except for the electrodes of the HCFL tube. You may have a question why the electrodes of the HCFL tube do not have the sine waveform. If the electrodes of the HCFL tube are electrically connected with the electron flow between the electrodes of the lighted FL tubes, the detected waveform should be the sine wave. But you detected the completely different waveform at the electrodes of the lighted HCFL tube. We cannot insertion of the electric resistance in the Ar gas space. You must detect the waveform at the electrodes of the FL tube.

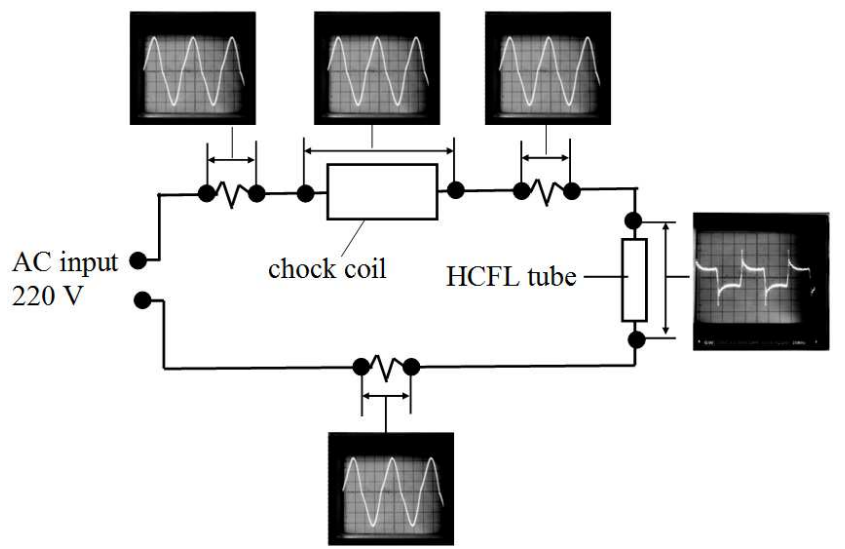

Figure 10. Waveforms of screen of oscilloscope at components and lead wires of commercial 40W-HCFL tube with ballast (choke coil).

The sine waveforms inform us that the AC driving circuit of the lighted HCFL tube at the electrodes of the FL tube is electrically closed with the AC current. The different waveform at the electrodes of the lighted FL tubes has assigned as the waveform of the "AC lamp current". As already described, the electrodes of the FL tubes do not inject the thermoelectrons into the Ar gas space. The waveform detected at the electrodes is not the waveform of the $\mathrm{AC}$ current in the Ar gas space. The waveform at the electrodes of the lighted FL tubes corresponds to the change in the voltage at the electrodes of the capacitor $\mathrm{C}_{\text {tube }}$ that is formed in the lighted FL tube. The reported and widely used AC current waveform is invalided in the study on the FL tubes.
The waveform at the electrodes of the lighted FL tubes is a most difficult item for the analysis. The reality of the different waveform is by the properties of the capacitor $C_{\text {tube }}$ that is formed in the Ar gas space in the lighted FL tubes [11]. We must have a good understanding of the physics of the $\mathrm{C}_{\text {tube }}$ of the lighted HCFL tube for the analysis of the waveform at the electrodes of the lighted FL tubes.

The sine waveforms at everywhere in the AC driving circuit indicate that the AC driving circuit is apparently shorted at the electrodes of the $\mathrm{C}_{\text {tube }}$. The electrically shorted electrodes of the $\mathrm{C}_{\text {tube }}$ gives the sine wave at the everywhere of the $\mathrm{AC}$ driving circuit shown in Figure 10. Then, we must find out how the electrodes of the lighted FL tube are apparently shorted in the AC operation of the $\mathrm{C}_{\text {tube }}$. The analysis of the $\mathrm{C}_{\text {tube }}$ is the different subject from this report. We will describe the details in other report.

\subsection{Wrong Determination of W of FL Tubes}

In the study on the FL tubes, we are facing a difficulty with the determination of the $\mathrm{W}_{\text {act }}$. Many commercial HCFL tubes indicate the $40 \mathrm{~W}-\mathrm{HCFL}$ tubes. The $40 \mathrm{~W}$ is not the real AC power consumption $\mathrm{W}_{\text {act }}$ of the external $\mathrm{AC}$ driving circuit. The $40 \mathrm{~W}$ is nominal power consumption. The nominal $\mathrm{W}$ is a large difference from the real $\mathrm{W}_{\text {act }}$. For the study on the $\mathrm{W}_{\text {act }}$ of the external AC driving circuits, we must clarify the definition of the $\mathrm{W}_{\text {act }}$ in the practice.

The AC driving circuits have two different power consumptions; apparent power consumption $\mathrm{W}_{\text {app }}$, and active power consumption $\mathrm{W}_{\text {act }}$. The AC driving circuits for the FL tubes always contain the impedance. The consumers who use the FL tubes pay their electric bill with the $\mathrm{W}_{\text {act }}$, instead of $\mathrm{W}_{\text {app. }}$. We take the $\mathrm{W}_{\text {act }}$ in this report.

Figure 11 illustrates the determined AC power consumption of the AC driving circuit of the commercial 40W-HCFL tube with the ballast (choke coil). The AC driving circuit contains two reactance that are (a) inductance composed by chock coil $\mathrm{L}$ and electric resistance $\mathrm{R},(\mathrm{L}+\mathrm{R})$, and (b) capacitance $\mathrm{C}_{\text {tube }}$ between the electrodes of the FL tube. AC input power is 220 $\mathrm{V}$ with $50 \mathrm{~Hz}$. The ordinary electric tester detects the AC current of $0.43 \pm 0.05 \mathrm{~A}$ at everywhere in the $\mathrm{AC}$ circuit. The reactance of the ballast is determined by the ordinal instruments in the laboratory. The ballast is composed with $\mathrm{L}$ $=4 \mathrm{H}$ and $\mathrm{R}=47 \Omega$. The reactance of the coil is calculated as $247 \Omega$. The voltage drop at the ballast is calculated as $106 \mathrm{~V}$ (= $247 \Omega \times 0.43 \mathrm{~A}$ ) that has confirmed by the ordinal tester. The $\mathrm{AC}$ active power consumption of the inductance is calculated as $46 \mathrm{~W}(=106 \mathrm{~V} \mathrm{x} 0.43 \mathrm{~A})$ that is the $\mathrm{W}_{\text {drive }}$. The capacitance of the $\mathrm{C}_{\text {tube }}$ in the lighted FL tube cannot determine by the ordinal instrument. The capacitance is calculated from (a) the AC voltage at the electrodes $(100 \mathrm{~V})$ of the HCFL tube, (b) AC current from the electrode $(0.43 \mathrm{~A})$ and (c) AC frequency (50 $\mathrm{Hz}$ ). The reactance of the $\mathrm{C}_{\text {tube }}$ is calculated as $232 \Omega\{=100 \mathrm{~V}$ $\left.\mathrm{x}(0.43 \mathrm{~A})^{-1}\right\}$. The capacitance that is calculated from reactance is $86 \mu \mathrm{F}\left\{=(232 \times 50)^{-1}\right\}$. The power consumption of the capacitance is calculated as $43 \mathrm{~W}(=100 \mathrm{~V} \times 0.43 \mathrm{~A})$ that is the AC power consumption of the lighted FL tube, $\mathrm{W}_{\text {tube. }}$ 


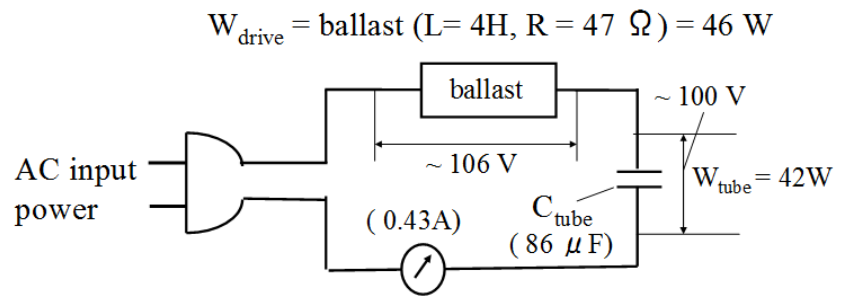

$$
\mathrm{W}_{\text {act }}=\mathrm{W}_{\text {tube }}+\mathrm{W}_{\text {drive }}=42 \mathrm{~W}+46 \mathrm{~W}=88 \mathrm{~W}
$$

Figure 11. Determined AC active power consumption $W_{\text {act }}$ of external $A C$ driving circuit of commercial $40 W-H C F L$ tube, and individual $A C$ power consumptions of $W_{\text {drive }}$ and $W_{\text {tube }}$ at driving parts.

From Figure 11, the AC driving circuit of the lighted FL tube consists with two different AC power consumptions, $\mathrm{W}_{\text {drive }}$ and $\mathrm{W}_{\text {tube }}$. Subsequently, the $\mathrm{W}_{\text {act }}$ is composed with sum of them; $\mathrm{W}_{\text {act }}=\mathrm{W}_{\text {drive }}+\mathrm{W}_{\text {tube }}$. The $\mathrm{W}_{\text {act }}$ is $89 \mathrm{~W}(=46 \mathrm{~W}+43$ $\mathrm{W})$. The electric power consumption of the commercial $40 \mathrm{~W}$-HCFL tube is actually $89 \mathrm{~W} \pm 5 \mathrm{~W}$. The $40 \mathrm{~W}$-HCFL tube is deliberately assigned with the $\mathrm{W}=\mathrm{W}_{\text {tube }}$ by neglecting of $\mathrm{W}_{\text {drive. }}$ The $40 \mathrm{~W}$ of the commercial $40 \mathrm{~W}-\mathrm{HCFL}$ tube is the nominal power consumption. The real $\mathrm{W}_{\text {act }}$ is given by $\mathrm{W}_{\text {drive }}+$ $\mathrm{W}_{\text {tube }}=89 \mathrm{~W}$. With $\mathrm{W}_{\text {drive }} \approx \mathrm{W}_{\text {tube }}$, the consumers who use the FL tubes as the light source pay their electric bill of the $\mathrm{W}_{\text {act }}$ that is the double cost of the nominal $\mathrm{W}=\mathrm{W}_{\text {tube }}$. Recently, the HCFL tubes are operated with the inverter with the frequencies higher than $30 \mathrm{kHz}$ with the main reasons of (a) rapid start of the FL tubes and (b) the prolonged operation life to 10,000 hours from 2,000 hours. The $\mathrm{W}_{\text {drive }}$ of the inverter is a half of the $\mathrm{W}_{\text {tube. }}$ The $\mathrm{W}_{\text {act }}$ with the inverter of the nominal $40 \mathrm{~W}$-HCFL tube is $\approx 60 \mathrm{~W}\left(=\mathrm{W}_{\text {drive }}+\mathrm{W}_{\text {tube }}\right)$ that is the 1.5 times of the nominal $\mathrm{W}=\mathrm{W}_{\text {tube }}$.

As described above, the nominal $\mathrm{W}$ of the commercial $40 \mathrm{~W}-\mathrm{HCFL}$ tubes is actually the $\mathrm{W}_{\text {tube }}$. Hence, the nominal $\mathrm{W}$ of the FL tubes invalids in the study on the FL tubes. We take the real $\mathrm{W}_{\text {act }}$ for the study on the lighted coil-EEFL tubes and nominal 40W-CCFL tubes.

\section{Conclusion}

We have studied the lighting mechanisms of the FL tubes with the different view points from the established technologies for 80 years, using needle electrodes. It has found the internal DC electric power generator formed in the Ar gas space by the aid of the electric field from the needle electrodes. The results lead us to the prototype of the coil-EEFL tube under the operation of the external DC driving circuit. We have found that the established FL technologies are inapplicable to the development of the coil-EEFL tube. Before the study of the details of the coil-EEFL tubes, we have clarified the invalided and inapplicable technologies of the FL tubes and PDP. The details of the development of the coil-EEFL tubes remain for the future study.

\section{Acknowledgement}

The author sincerely wishes to express his appreciation to Dr. Takao Toryu for his instruction of the project.

\section{References}

[1] J. F. Waymouth, "Electric Discharge Lamp", MIT Press, 1971

[2] Handbook of "Electric Discharge lamps", Japanese Institute of Electric Engineers, 1973

[3] Lyuji Ozawa, "Illuminance $\left(1 \mathrm{~m} \mathrm{~m}{ }^{2}\right)$ of compact 20W-HCFL tube", Science Research, 3, pp 170-179, 2015.

[4] American Vacuum society Classics, (1) The fundamental data on electrical discharge gases, (2) Field emission and field ionization, (3) Vacuum technology and space simulation, (4) The physical basics of ultrahigh vacuum, (5) Handbook of electron tube and vacuum techniques, (6) Vacuum sealing techniques, and (7) Ionized gases, American Institute of Physics, 1993

[5] L. Ozawa and Y. Tian, "calculation of quantum efficiency of phosphor screens in CRTs and FL tubes, Korean J. Information display, 11, pp 128-133, 2010

[6] Encyclopedia Chimica, volume 7, p 25, 1969, Kyoritsu Publishing Co. Japan

[7] F. A. Kroger, "Some aspect of the luminescence of solids", Elsevier Pub. Co., New York, ’1948

[8] H. W. Leverentz, "An introduction of luminescence of solids", John Wiely\& Sons Inc., London, 1950

[9] L. Ozawa, "Cathodoluminescence, Theory and application" Kodansha Ltd, Tokyo, Japan and VCH Publishers, UK, 1990

[10] L. Ozawa, "Cathodoluminescence and Photoluminescence, Theory and practical application, CRC Press, Taylor \& Francis Group, Boca Raton, London, New York, 2007

[11] L. Ozawa and Y. Tian, [A new 4G electron source for Fluorescent Lamp tubes], J. China Ill. Engs. Soc., 7, 58-65, 2012

[12] L. Ozawa and Y. Tian, "Coexistence of disparities of external AC driving circuit and internal DC electron circuit in operation of fluorescence tube", J. China Ill. Engs. Soc., 6, 19-30, 2011. 